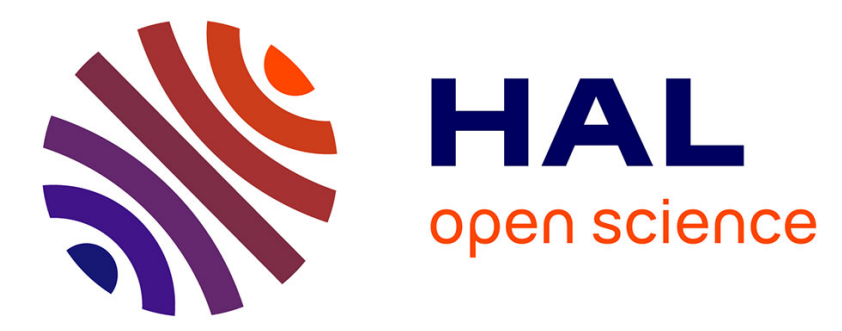

\title{
L'innovation organisationnelle sous les tensions performatives. Propositions pour l'analyse d'une co-construction conflictuelle des politiques et pratiques numériques \\ Maryse Carmes
}

\section{To cite this version:}

Maryse Carmes. L'innovation organisationnelle sous les tensions performatives. Propositions pour l'analyse d'une co-construction conflictuelle des politiques et pratiques numériques. Les Cahiers du numérique, 2011, Piloter l'entreprise à l'ère du numérique, 6 (4), p.15-36. 10.3166/lcn.6.4.15-37 . hal-00819016

\section{HAL Id: hal-00819016 https://hal.science/hal-00819016}

Submitted on 29 Apr 2013

HAL is a multi-disciplinary open access archive for the deposit and dissemination of scientific research documents, whether they are published or not. The documents may come from teaching and research institutions in France or abroad, or from public or private research centers.
L'archive ouverte pluridisciplinaire HAL, est destinée au dépôt et à la diffusion de documents scientifiques de niveau recherche, publiés ou non, émanant des établissements d'enseignement et de recherche français ou étrangers, des laboratoires publics ou privés. 


\title{
L'INNOVATION ORGANISATIONNELLE SOUS LES TENSIONS PERFORMATIVES ${ }^{1}$
}

\section{Propositions pour l'analyse d'une co-construction conflictuelle des politiques et pratiques numériques}

\author{
MARYSE CARMES²
}

Cet article interroge les politiques et stratégies numériques des organisations et plus particulièrement la fondation d'un modèle assis sur le déplacement au sein de l'entreprise, des dispositifs 2.0 ou «social software». À partir de la notion de performation et de travaux de l'anthropologie des sciences et techniques, la perspective d'un agencement d'innovation complexe, hétérogène et traversé de conflits, est développée. L'enquête réalisée au sein de deux grandes entreprises publiques françaises, permet de mettre en évidence les confrontations qui ont cours entre une performation théorico-doxique, une performation machinique, une performation expérimentale, une performation expérientielle et enfin une performation désirante. Deux axes majeurs de débats, associés à l'extension des pratiques socio-numériques des salariés, sont isolés : la «pression » des intranautes-internautes dont la montée en compétences s'accompagne d'une montée en exigences vis-à-vis de l'entreprise; l'extension des territoires d'individuation professionnelle et la gouvernance des frontières info-communicationnelles normatives.

Quelques mois seulement séparent la prescription de la Work Fondation ${ }^{3}$, en faveur d'une adoption d'un «social software » au sein des organisations, et la vulgarisation de la notion de « web 2.0 » par Tim O'reilly ${ }^{4}$. En 2007, le célèbre cabinet américain Gartner proclame que «l'ère de l'entreprise 2.0 est

\footnotetext{
1 Version révisée de Carmes M, «L'innovation organisationnelle sous les tensions performatives : Propositions pour l'analyse d'une co-construction conflictuelle des politiques et pratiques numériques ", in Debos F. (dir), Piloter l'entreprise numérique, Les Cahiers du Numérique, Hermès-Lavoisier, n 4/2010, p.15-36

2 Maitre de Conférences au CNAM-INTD, Laboratoire DICEN-IDF

3. You don't know me but...Social capital and social software, Work Fondation, institut britannique regroupant chercheurs et praticiens, janvier 2003.

4. L'expression «social software» est entrée en circulation dans le courant de l'année 2002 aux USA, pour caractériser une croissance significative des applications «orientées utilisateur» sur internet et leurs dimensions sociales, mais elle a véritablement été lancée, dans les réseaux technico-économiques, en avril 2003, lors du colloque «O'Reilly Emerging Technology Conference» tenu à Santa Clara. En 2005, la notion de "web 2.0 » lui semble préférée et est instituée par Tim O’Reilly. O’Reilly, T. 2005: What is Web 2.0 ? [en ligne]. http://oreilly.com/web2/archive/what-is-web-20.html. Loin d'une stabilisation, on assisterait aujourd'hui à certain
} 
venue ${ }^{5}$ et les prévisions économiques, qui sont attachées à cette industrie du «web social», que ce soit sur internet ou en entreprise, nous sont présentées aujourd'hui comme un marché à très fort potentiel 6 . Ainsi, le déplacement, au sein des organisations, des dispositifs socio-numériques d'internet (blogs, wikis, réseaux sociaux numériques etc.) semble s'accompagner d'une emphase illocutoire et marketing sans limites. En France, les colloques et ouvrages se sont largement mobilisés sur le sujet et les porte-parole «visionnaires» ne cessent de formater la perception des temps socio-techniques, du couplage technogenèse-sociogenèse (Stiegler, 1996), en prônant le modèle archétypal que constitue «l'organisation $2.0 »$. Promesses déterministes et généalogies normatives des intranets, célébrations des «bonnes pratiques » portées par des instances associatives ou médiatiques (Carmes, 2009), mots d'ordre sur la rupture des stratégies managériales et info-communicationnelles : tout cela contribue à construire un caractère d'urgence. En suivant les travaux réalisés par M. Callon et B. Latour sur le capitalisme, qui l'analysent comme résultat de processus performatifs, nous pouvons convenir que nous avons ici affaire à un milieu construisant «la théorie de sa propre pratique et la pratique de sa propre théorie » et procédant au «formatage de son propre formatage» (Callon, Latour, 1999). Se pensant auto-fondateur et autolégitimant, le monde organisationnel est habité par la création continuée de lui-même.

Or, les stratégies et pratiques estampillées «social software » ou 2.0, dans le cadre de leur intégration dans un territoire numérique normatif (les intranets), restent encore fortement discutées. Derrière le consensus apparent, et au sein des directions et équipes projets, s'exprime un vif débat sur l'adoption des solutions 2.0 et des scripts socio-techniques qui les accompagnent. Ce débat se trouve massivement suscité et contraint par l'exposition libre de salariés et de communautés professionnelles sur des réseaux sociaux numériques, des blogs ou des forums de discussions (l'usage de ces derniers perdure). Si les pratiques socionumériques qui ont cours sur la toile et les transformations anthropologiques qu'elles portent s'avèrent profondes et très activement étudiées (cf par exemple : Millerand F. et al. 2010 ; Stiegler et al., 2010 ; Stenger, Coutant, 2010 ; Licoppe et al., 2009; Moatti, 2009; Allard, 2007 ; Boyd, Ellison, 2007), elles constituent ici une opportunité pour interroger une négociation politique de prescriptions aux accents doxiques.

L'innovation comme problème consiste alors à examiner comment elle se produit et se négocie à partir de la composition complexe de processus performatifs. Nous menons notre analyse à partir des travaux de l'anthropologie des sciences et des techniques conduits dans le champ économique (Muniesa, Callon, 2008), mais nous adaptons leurs apports à notre objet. En mobilisant la notion de performation, nous insistons sur l'agencement hétérogène à partir duquel émerge et s'incarne " une fabrique de l'organisation 2.0 », et ce dès les débats qui la traversent et lui donnent chair. Cette performation est envisagée en tant qu'activité distribuée, reposant non seulement sur les narrations, les agencements collectifs d'énonciation, avec leurs stratégies discursives, leurs diagrammes prescriptifs et les diverses inscriptions-supports qui en permettent la dissémination, mais aussi sur les pratiques concrètes et les technologies mobilisées. Il convient, en effet, de prendre en compte une performation non exclusivement ramenée aux "discours », en intégrant d'emblée l'action des objets, des interfaces machiniques (Guattari, 1991 ; Lazzarato, 2006), et les pratiques hétérogènes qui leur sont associées. Bref, il s'agit de prendre la mesure des divers types de performation en lutte à l'occasion de ce procès que l'on nomme innovation.

délaissement de l'expression « $2.0 »$ (chez les grands cabinets comme Gartner ou Forrester), le web 3 et le web-cube étant déjà annoncés.

5. Prédiction de 2007, sur le social software et l'entreprise en janvier 2010 : US\$1,995.00, in «Predicts 2010: Social Software Is an Enterprise Reality ", http://www.gartner.com/technology/analysts.jsp

6. Prédiction de 2008 : "Enterprise 2.0 will become a $\$ 4.6$ billion industry by 2013 and social networking tools will garner the bulk of the money, according to a report by Forrester Research.” http://www.forrester.com/rb/Research 16 
Dans cet article, nous proposons d'examiner les tensions performatives auxquelles se confrontent le management, et plus particulièrement, celles attachées aux pratiques et expériences socio-numériques des salariés. Nous exploitons deux cas d'organisations en mettant en évidence les rapports de force entre une performation théorico-doxique, une performation machinique, une performation expérimentale et une performation expérientielle. Plus globalement, on montre que la question politique hante plus que jamais, sous des incarnations nouvelles et des affrontements inédits, la création de stratégies numériques en se constituant en véritable économie politique « des désirs » et de la raison innovatrice.

\section{La performation des stratégies numériques d'organisations}

\section{L'agencement performatif et son questionnement}

Dans les recherches portant sur la communication en milieu organisationnel, la question performative s'est éminemment centrée sur l'analyse des narrations, des sémiotiques de l'organisation et au façonnage de cette dernière par les interactions, les récits et les écritures sous leurs diverses formes, qu'elles soient ici chartes, normes, journaux internes, systèmes de gestion etc. (Grosjean, Lacoste 1998; Dalmeida, 2001 ; Frankael, 2006 ; Gryspeerdt, Marion, 2002 ; DeLaBroise, Grosjean, 2010). Or, à certains égards dans les travaux sur les organisations, la perspective a souvent maintenu une dissociation de «l'endogène/exogène », des dedans/dehors, quand, comme le souligne F.Cooren, il s'agirait d'envisager les modes d'être des organisations et des processus organisants, en réassemblant le local et le global (Latour, 2006 7) et ce, afin de pouvoir aborder ces questions d'un point de vue à la fois constitutif et performatif (Cooren, 2010).

Développé encore plus radicalement par l'anthropologie des sciences et des techniques, l'examen des processus performatifs a notamment suscité un vif intérêt en sociologie économique. Introduit dans ce champ par Michel Callon dans ses travaux sur la performativité des sciences économiques (1998), le « concept» de performativité (ou de performation) est aujourd'hui largement reconnu par de nombreux auteurs et ses implications y sont débattues (cf par exemple, Economy and Society, 2002 ; Journal of Cultural Economy, 2010). Ce phénomène, objet pluridisciplinaire, nous oblige à repenser les politiques et dispositifs organisationnels, leur élaboration, à partir de l'intervention d'actants ${ }^{8}$ hétérogènes, en opérant non seulement " une décentration du tout langagier » (tel que proposé par rapport aux processus normatifs en organisation; De LaBroise, Grosjean, op. cit.), mais aussi, en restituant aux actants matériels leur pleine puissance. A l'instar de M. Callon et F. Muniesa, nous préférons l'emploi de la notion de «performation » à celle de "performativité » ou "d'énoncé performatif» forgée par J. L. Austin (1970), afin de nous extraire, autant que faire ce peut, de l'emprise du langage, et privilégier la prise en compte d'une narratique non exclusivement ramenée aux discours ou aux écrits, en intégrant les conditions politiques, sociales et techniques qui participent, en associant plusieurs niveaux d'échelle, à la création d'un référentiel d'actions et au formatage des ensembles technico-organisationnels.

\footnotetext{
7. Selon B.Latour, il s'agit de mener à bien la tâche qui consiste à assembler le collectif, mais seulement après avoir abandonné les raccourcis de la "société 》 comme l'explication du social et de considérer que le macro n'est ni au-dessus ni en-dessous des interactions : il vient s'ajouter à elles comme une autre connexion qui les alimente et qui s'en nourrit. (Latour, 2006, p. 259).

8. Le concept d'actant se réfère notamment aux travaux de la sémiotique et de la sociologie de la traduction, désignant par là les instances humaines et "non humaines » présentant une capacité à affecter ou à être affecté. Préféré à ceux d'acteur ou d'agent, utilisés par les sociologues, le terme actant permet de recouvrir non seulement les êtres humains, mais aussi les objets, les théories. Un actant correspond à toute entité (technique ou non), tout intermédiaire, déclencheur d'action.
} 
Bien sûr, l'activité discursive est ici essentielle, mais encore s'agit-il de prendre les discours sur les usages des TIC «dans leur pleine et entière positivité, non pas comme des discours d'accompagnement, mais comme éléments constitutifs de la "machine collective » qui donne sens à l'élément technique. Ils performent le monde et participent à notre auto-expérimentation, symbolique, imaginaire, réelle » (Noyer, 2006). Ces discours et récits organisent une composition de nombreux objets techniques et donnent réalité à ces «choses» qui «n'existaient pas» il y a encore quelques années : «l'e-organisation", son prolongement « l'organisation 2.0 », et tous les dispositifs, ingénieries, marchés, acteurs, objets, pratiques qui vont alors s'y rattacher. Actants humains et non humains s'agencent et participent au processus de performation dans des espaces socio-techniques, des réseaux socio-cognitifs. Pour G. Deleuze (proposant une autre perspective du dispositif de M. Foucault), l'agencement est, de manière indissoluble, «à la fois agencement machinique d'effectuation et agencement collectif d'énonciation ». À ce titre, ce dernier règle sous divers modes (non exclusivement linguistiques), la production et la distribution des énoncés, « ce qui se dit et s'échange » : «L'énoncé est le produit d'un agencement, toujours collectif, qui met en jeu, en nous et en hors de nous, des populations, des multiplicités, des territoires, des devenirs, des affects et des événements» (Deleuze, Parnet, 1977/1996, p. 65). L'agencement, dont les règles d'analyse sont rassemblées par G. Deleuze et F. Guattari dans la «conclusion » de Mille Plateaux, met en jeu une pensée de la relation, de la connexion et des compositions de rapports «qui font tenir ensemble ces hétérogènes ». L'agencement se définit alors notamment par les «alliances ", «alliages », "attraction et répulsion », "sympathie et antipathie», "altération» etc. qu'il facilite ou censure, et donc aussi par le potentiel de transformation qu'il permet. Il ne s'agit plus ici de poser le problème en termes de diffusion des technologies, pratiques, doxas etc. à partir de la présupposition d'un centre, mais de considérer les dynamiques, les connexions qui s'agrègent, les rapports de forces qui s'établissent entre une pluralité d'acteurs et de localités. La performation devient ainsi «un ensemble d'activités et d'événements qui instaurent ou modifient un agencement» (Callon, Muniesa, op. cit.).

Enfin, le questionnement de la performation dépasse le seul objectif de savoir si tel modèle, tel script socio-technique, sont effectivement suivis et réalisés à lettre, ou si telles théorie ou "prophétie» rencontrent les conditions de félicité nécessaires à son auto-réalisation effective (Merton, 1949) 9. À notre sens, la performation relève aussi «du circuit virtuel/actuel» ou, pour reprendre le vocabulaire de G. Deleuze, d'une «cristallisation» (Deleuze, 1968) ${ }^{10}$. Selon P. Levy est virtuel ce qui existe en puissance et non en acte. []. En toute rigueur philosophique, le virtuel ne s'oppose pas au réel mais à l'actuel: virtualité et actualité sont seulement deux manières d'être différentes. (Levy, 1995). En ce qui concerne les technologies social software, déjà disponibles sur internet, elles portent des virtualités qui, dans le monde organisationnel, vont s'incarner selon des formes variées. Toute décision prise quant au déploiement de technologies 2.0 au sein d'une organisation (les intégrant ou les rejetant), toute pratique professionnelle des salariés qui les utilisent « hors des murs» de celle-ci, tout programme et logiciel de réseaux sociaux d'entreprise proposés par des éditeurs, les scripts socio-techniques, les débats et passions que tout cela suscite, actualisent des formes organisationnelles qui ne cessent en même temps de se différencier des finalités annoncées et des buts affichés. Les performations qui expriment ces actualisations sont des procès complexes et en tension.

9. Pour Callon, le succès d'un modèle et la véracité de ses déclarations ne se distinguent pas.

10. Le virtuel dans la pensée de Deleuze, est rigoureusement distinct du possible. L'actuel et le virtuel composent la réalité comme ses deux moitiés asymétriques. 


\section{Les configurations performatives}

Une pluralité de configurations performatives ont été décrites dans les travaux conduits par F. Muniesa et M. Callon. Afin d'appréhender les rapports de force et les tensions qui traversent l'agencement performatif qui nous intéresse ici, nous tentons une mobilisation des propositions de ces deux chercheurs, à notre objet, tout en soulignant les difficultés du cadre théorique utilisé. Premièrement, les auteurs appréhendent l'articulation entre une "performation théorique » et une "performation expérimentale » (rattachée aux situations d'ingénierie, aux activités des équipes projet). La première se caractérise par des configurations dans lesquelles l'enjeu principal est de construire un monde à l'image d'une théorie, c'est-à-dire de verser sur un monde un ensemble de problèmes et solutions exprimés préalablement de manière abstraite dans un corps théorique. À bien des égards, l'examen d'une performation, mobilisant des travaux de sociologie, de sciences de gestion ${ }^{11}$ ou des sciences de la communication, et opérant sur des rationalités organisationnelles (qui peuvent fonctionner en aveugle par rapport à ce qui les structure) ainsi que sur des usages (contraints par des programmes et interfaces des plate-formes logicielles), ouvre des problématiques de recherche stimulantes ${ }^{12}$. Toutefois, celles-ci engagent un travail ethnographique et de récolte de "traces» éminemment conséquent, voire aléatoire: par exemple, le récent exercice d'une association sur la communication interne, tentant une étude sur la prégnance des sciences humaines sociales à partir d'entretiens auprès de directions de grandes organisations, est à bien des égards trop limité.

La «théorie » à laquelle on se réfère dans cet article est à considérer de manière plus restreinte : elle ne se situe pas au niveau académique mais dans le domaine opérationnel (associations et presses professionnelles, experts, conseillers d'entreprise, chefs de projets, organismes d'études etc.), donc plutôt du côté des contraintes dogmatiques, des prescriptions générales et normatives, qui tendent à définir un modèle info-communicationnel et organisationnel, assis sur les paradigmes du « web 2.0 ». Le résultat de cette performation, que l'on peut qualifier de théorico-doxique, décrit un monde organisationnel et contribue à le réaliser. Elle s'incarne dans une codification collective des connaissances, dans les référentiels d'action rendus disponibles et institués par divers leaders d'opinion. Les opérations de "problématisation», le "processus d'intéressement», d'enrôlement et de «mobilisation des alliés » (selon la perspective de la sociologie de la traduction), vont impliquer de nombreuses instances-intermédiaires, parmi lesquelles on retrouve des associations professionnelles, des «concours ${ }^{13}$, des cabinets conseils, des ouvrages professionnels et autres articles de presse. Sur la période de janvier 2003-mars 2010, et relativement aux acteurs spécifiques que constituent les revues professionnelles, nous avons recensé plus de 800 articles (d'origine française) concernant l'usage des RSN par les salariés avec une croissance très forte à partir de 2008 (80 \% des articles sont produits à partir de cette date). «L'intranet 2.0 » devient un thème émergent abordé dans la presse d'entreprise ou généraliste à partir de $2006^{14}$ et, dans le même temps, plusieurs

11. Une approche critique des sciences de gestion a été récemment relancée en ce sens. «Elle questionne la nature et les finalités des connaissances produites dans la discipline, dénonçant le "managérialisme" de travaux de recherche qui adoptent implicitement les finalités managériales et ce faisant les légitiment en leur conférant la neutralité d'un statut technique et scientifique. » voir la conférence AIMS 2010, Luxembourg et Palpacuer, et al, 2010.

12. Pour les seuls réseaux sociaux numériques (Facebook et autres), il serait utile de ré-interroger la mobilisation explicite de la théorie des graphes, des travaux de Milgram ou encore de Granovetter, dans leurs modèles, configurations et discours de légitimation.

13. Le prix Intranet organisé par Entreprise \& Carrières/Les Echos/Cegos, depuis 1998, en est une illustration. Ce dispositif porte l'autoconviction de sa puissance performative. Nous avions notamment étudié ce processus dans notre travail de thèse.

14. Sélection: «L'électronique de loisirs se décline dans le monde du travail », Les Échos, N¹9586 du 18 Janvier 2006 ; «BlueKiwi met les entreprises au Web 2.0 »; l'Expansion, 25/05/2007 ; « Le travailleur nomade fait sa migration 
ouvrages à destination des managers, s'emparent du sujet ${ }^{15}$. C'est aussi une période faste pour l'organisation de colloques et séminaires professionnels souhaitant promouvoir cet « intranet du futur » ${ }^{16}$. Tant Lyotard que Bourdieu, ont rappelé que la position sociale, l'autorité, le pouvoir dont est doté l'énonciateur, étaient des conditions essentielles de félicité d'un énoncé performatif (Lyotard, 1979; Bourdieu, 1982). Ce n'est donc pas la qualité intrinsèque de l'innovation socio-technique qui fait qu'elle va ou non s'imposer, mais la valeur dont se trouvent investis un ensemble d'intermédiaires-médiateurs parlant au nom d'autres entités. La performation théorico-doxique se trouve ainsi étroitement mêlée, dans notre cas, à ce que Callon et Muniesa dénomment performation psychogène et qu'ils rattachent aux croyances, aux représentations collectives, ou encore aux expériences psychiques. À ces notions, nous préférons la perspective d'un agencement traversé de subjectivités, d'intentions, de stratégies, de forces, de passions, bref, de désirs (au sens de production sociale et désirante telle que pensée par G. Deleuze et F. Guattari), alimentant alors une techno-politique des projets intranets dits 2.0. Nous désignons ce processus par le terme de performation désirante. Elle mobilise une multiplicité de mots d'ordre, slogans, diagrammes et indicateurs (les porte-parole administrent les éléments de "preuve» et les appareils d'évaluation). Tout cela participe au creusement des «chréodes temporelles et stratégiques» (Noyer, 2002), des points de passages obligés de l'action organisationnelle, et nourrit un désir de "révolution » (qui n'en reste par moins soumise au phantasme de contrôle) sans cesse relancé.

Le troisième mouvement, une "performation expérimentale », se réfère "aux situations d'ingénierie dans lesquelles la démarche part de problèmes et de situations problématiques pour élaborer progressivement les modèles, mesures et instruments économiques qui, mobilisés pour transformer ces situations problématiques, pourraient apporter des solutions aux questions posées. » Responsables et décisionnaires d'entreprises, dont nombre d'entre eux participent directement aux productions « théoricodoxiques ", formulent et prescrivent des stratégies qui sont le résultat d'un processus réflexif sur leurs contingences et contraintes propres à leur organisation. Même si certains peuvent exposer des pratiques très innovantes et singulières, en recherche de valorisation, les acteurs seront souvent en démonstration de leur conformisme par rapport aux modèles institués. Par ailleurs, tel que nous l'avons montré dans une recherche portant sur la conception d'un dispositif de gestion collaborative des ressources humaines (cas Moeva, Carmes, 2008), les scripts socio-techniques (ce dont il convient de décider, les justifications, les contraintes et les effets attendus de cela), leurs cahiers des charges, ainsi élaborés au sein d'un groupe projet, vont pouvoir se déplacer d'un lieu en d'autres lieux. Dans la sociologie de la traduction, "pour qu'ils deviennent de «véritables » performatifs, les faits, les théories ou les formules doivent circuler dans des chaînes de traduction qui consolident l'assemblage des entités qui le composent et leur permet

vers le «mobile 2.0 », Les Échos, 21/05/08; «Le Web 2.0, un outil de gestion RH », Silicon.fr, 12/06/2008; «Les services RH se mettent au Web $2.0 »$, LEntreprise.com, le 28/04/2009; "L'utilisation des outils Web 2.0 est encore timide dans l'entreprise ", La Vie Éco, 3 août 2009 ; "Comment les entreprises apprivoisent le Web 2.0 », Les Échos, 13 oct. 2009 ; «Les réseaux sociaux raniment les intranets », Usine Nouvelle, 26 mars 2010.

15. Un précurseur: L’Art du Management 2.0 , PriceWaterHouseCoopers, Les Échos, et Le Financial Times (coll), 2001 ; F. Nonnenmacher, Blogueur d'entreprise, éds d'Organisation, 2006 ; Martin Roulleaux-Dugage ; Organisation 2.0 : Le knowledge management nouvelle génération, éditions d’Organisation ; 2007 ; G. Hamel, B. Breen La Fin du management : Inventer les règles de demain, Vuibert, 2008; F. Créplet et T. Jacob, Réussir un projet Intranet 2.0, éditions d'Organisation, 2009 ; B. Jarrosson (Auteur), P. Kosciusko-Morizet (Préface), Vers l'économie 2.0 : Du boulon au photon...!, Les Échos, eds d'Organisation, 2009.

16. «De l'intranet traditionnel aux perspectives de l'internet 2.0 », Intracom, Paris, avril 2006 ; «Intranet 2.0 : L'ère du Peopleware : retour à l'utilisateur et e-transformation », Intracom, Paris, avril 2007 ; «De la révolution dans l'air ; web 2.0 état de l'art », Intracom, Paris, mars 2008, «Intranet 2.0 et e-Transformation, » Intracom, Paris, mars 2009 , « Entreprise 2.0 Forum : première édition parisienne », 17 et 18 mars 2010, à l'initiative de Bertrand Duperrin... 
d'acquérir le statut de matters of fact (Denis, 2006). De même, «ce transport» sera favorisé (en sus des porte-parole évoqués plus haut) par les articulateurs spécifiques que constituent les éditeurs et concepteurs de solutions technologiques : ils capitalisent les développements réalisés pour un client auprès de plusieurs autres organisations clientes qui peuvent alors adopter et adapter les «standards » du premier. Les scripts socio-techniques opèrent telle une « capture » recomposant des singularités, des différences, des stratégies et des croyances locales (Carmes, op. cit. 2008).

Nous nous engageons alors dans un quatrième type de mouvement de performation, souligné par les deux chercheurs, qui dans le contexte des TIC, devient tout à fait remarquable : il s'agit de questionner la performation machinique (Callon et Muniesa emploient le qualificatif «matérielle ») résultant de l'agissement des objets techniques sur les subjectivités et les comportements. L'artefact peut être à la fois pris, dans une performation d'après-coup (leurs inventeurs peuvent se préfigurer un monde social, mais tous n'attendent pas «que le sociologue leur définisse la société dans laquelle ils vivent» (Latour, 1984, p. 67), et dans un processus de performation adossé à un "programme d'action » (Akrich, 1993). Dans notre perspective, et à la suite d'un travail initié avec J.M. Noyer (Noyer, Carmes, 2010), nous avons affaire à des «interfaces machiniques » à partir desquelles les processus de performation tentent de frayer leur voie : elles sont équipées de dispositifs de filtrages, de droits, de règles de relations, d'indexations, de recherche, de cartographies, de systèmes d'annotation, de logiciels et d'écritures hypermédias diverses, qui se constituent en «milieux » où se définissent et s'alimentent les pragmatiques internes et externes des machines sociotechniques, des semiopolitiques organisationnelles.

Ces interfaces machiniques entrent en couplage avec le cinquième mouvement que nous proposons d'étudier ici, à savoir la performation expérientielle: elle se réfère pour nous, aux situations où la négociation des modèles s'effectue à partir des expériences et pratiques numériques des salariés (l'open source et son enrichissement à partir des développements et recommandations des utilisateurs pourrait en être l'un de ses pendants sur internet) : il s'agit de voir comment, via leurs apprentissages (d'internet et intranet) et les compétences alors acquises, les salariés deviennent eux-mêmes intercesseurs potentiels des transformations organisationnelles en cours, et comment ce processus pourrait entrer en conflit éventuel avec les autres processus de performation.

Ainsi, ces cinq forces performatives de «la fabrique de l'e-organisation», théorico-doxique, machinique, expérimentale, expérientielle et désirante, vont pouvoir se déployer au sein d'une multiplicité de relations, antagonistes ou complémentaires, simultanées ou asynchrones. Elles s'enchevêtrent et participent à l'instauration d'un agencement, se distinguent en fonction de l'intensité des tensions qui s'expriment entre elles.

\section{Les pratiques socio-numériques des salariés : l'affrontement de l'organisation à une performation expérientielle}

\section{Méthodologie et terrain de recherche}

Dans les organisations, l'espace normatif, contrôlé, que constitue l'intranet, s'appuie sur des interfaces machiniques chargées de poser ce en quoi consiste le territoire numérique professionnel des salariés. Répondant à un besoin de « localiser » (un « soi », un « nous » par rapport à autrui), le territoire s'écrit par ses frontières, par ses "marquages", par ses inscriptions (que peuvent être les ergonomies) ou encore à travers la définition «autoritaire» de listes de sites internet autorisés (« un dehors » que l'on pourrait consulter à partir de son travail), des ressources informationnelles jugées comme légitimes (sites de filiales, partenaires, clients, presse, cotation boursière affichée sur la page d'accueil des grands groupes privés...). 
Dans ce cadre, les annuaires LDAP ${ }^{17}$, à partir desquels sont gérés les divers droits des utilisateurs (autorisation d'accès à telle rubrique ou application, autorisation de contribution ou de modification, administration d'un espace etc.), sont aussi des actants privilégiés du formatage des pratiques: le userprofiling n'est autre qu'une projection-prescription de la « situation » de l'utilisateur ideal-typé. Construction collective, sociale et politique, le territoire est le produit d'un acte de territorialisation, de "surcodage » pour reprendre le vocabulaire de G. Deleuze et de F. Guattari (Deleuze, Guattari, op. cit.). Tel que nous l'avons déjà exposé (Noyer, Carmes, op. cit.), les sémiotiques signifiantes "performent» ici l'ordre des identités et des rôles, des êtres, des systèmes hommes-machines, des territoires, et entrent en de complexes mélanges avec des sémiotiques a-signifiantes enchâssées dans les applications, les programmes et développements logiciels qui sont proposés. Inhérente aux stratégies des concepteurs et responsables projets, la performation machinique qui se déploie ainsi est au cœur des relations savoir/pouvoir. Nous souhaitons l'étudier dans sa relation, sa tension à la performation expérientielle, performation liée aux pratiques numériques des salariés. Cette relation constitue un des débats majeurs des politiques numériques d'entreprise.

Pour ce faire, nous exploitons deux cas de grandes organisations et analysons dans ce cadre les tensions inhérentes au développement des usages socio-numériques d'internet par les salariés. Pour chacune des organisations, nous avons développé une approche ethnographique permettant de mettre en rapport les perceptions des salariés et des équipes de directions ou projets TIC. De plus, une méthodologie spécifique a été adoptée afin d'obtenir des données sur les pratiques salariés. Pour le cas Angus, nous exploitons les résultats d'une enquête en ligne, auto-administrée (seuls certains résultats en rapport avec notre sujet sont présentés ; voir Carmes, Galibert, 2010) ; pour le cas Karina, nous avons procédé à une analyse de communautés de salariés sur internet ${ }^{18}$. Dans l'entreprise Angus, nous mettons en exergue les tensions relatives à la montée en compétences des salariés et à la redistribution des expertises info-communicationnelles, et pour ce qui est de Karina, c'est la perception d'une "guerre des territoires » qui est ici analysée.

\section{La montée en compétences et en exigences des salariés}

Diverses études tendent à montrer que les dispositifs et ressources technologiques, ainsi que les usages associés, seraient aujourd'hui globalement moins innovants dans la sphère professionnelle que dans la sphère privée ${ }^{19}$ (il faut évidemment relativiser ce constat au regard de l'ancrage des dispositifs numériques dans les entreprises à dominante technologique, dans le monde de la recherche etc.). Ce décalage résulterait de l'avance technologique d'internet sur les intranets d'entreprises mais aussi du développement des dimensions collectives et sociales qui sont permises par le premier et très peu par les seconds

15. Lightweight Directory Access Protocol (LDAP); terme utilisé pour désigner un protocole et un serveur d'annuaire. Celui-ci répertorie ici les utilisateurs d'un intranet et permet la gestion des identités, des mots de passe et des droits associés à chacun. Cet annuaire permet aussi de simplifier la gestion des utilisateurs en ne leur demandant qu'une seule authentification (SSO : Single-Sign-On) pour accéder aux applications, services et systèmes de l'entreprise.

18. Cas Angus : la recherche a été réalisée de janvier à juin 2009, au sein d'une grande organisation publique française regroupant plus de 140000 salariés (en majorité des techniciens et agents de service à la clientèle). L'enquête en ligne auto-administrée, exploite les données de plus de 9000 répondants. Cas Karina : la recherche, toujours en cours, a été initiée en juillet 2010, au sein d'une seconde organisation publique de plus de 200000 salariés. 12 réseaux et communautés de salariés ont été analysés. Dans les deux cas, plusieurs entretiens semi-directifs, sur le lieu de travail, ou de groupes ont été réalisés (directions, managers et non cadres). Seuls certains résultats sont présentés ici.

19. Tic et Entreprise -Insee Première, N¹184, avril 2008 ; étude Ipsos, novembre 2008. 
(beaucoup d'intranets n'ont pas adopté «le référentiel $2.0 »)$. Cela est le cas de l'organisation Angus étudiée ici. Les salariés ne perçoivent que très faiblement le positionnement «collaboratif» de l'intranet (on note par exemple l'absence d'espaces partagés métiers, d'outils de veille partagée, d'outils de recherche et de partage efficace des ressources) et il en est de même pour sa contribution à l'enrichissement du dialogue social. Il convient évidemment de distinguer la perception des utilisateurs experts, intensifs, de celles des utilisateurs occasionnels et des non-utilisateurs (subissant une impossibilité de connexion à l'intranet ou affirmant un rejet volontaire) ${ }^{20}$. Le tableau 1 n'indique que les résultats généraux relatifs aux salariés utilisateurs.

Seuls $25 \%$ des salariés pensent que c'est un moyen d'échanges avec les collègues (les rares forums qui existent sont peu utilisés et $85 \%$ n'utilisent jamais les outils interactifs de l'intranet). Il se positionne faiblement en tant que dispositif de capitalisation des savoirs collectifs (peu de valeur ajoutée perçue en matière d'identification des personnes ressources et de mutualisation des compétences et savoir-faire).

Tableau 1. Positionnement attribué à l'intranet Angus selon les salariés utilisateurs 21

\begin{tabular}{|l|c|}
\hline Un moyen d'échanges avec ses collègues & $25 \%$ \\
\hline $\begin{array}{l}\text { Une ressource pour mieux connaittre, exploiter et mutualiser } \\
\text { les compétences et les savoir-faire }\end{array}$ & $23 \%$ \\
\hline Un espace offrant de nouvelles conditions d'échanges et de débats & $20 \%$ \\
\hline $\begin{array}{l}\text { Un outil qui permet de mieux identifier les compétences } \\
\text { et les personnes }\end{array}$ & $14 \%$ \\
\hline Un moyen pour créer et enrichir le dialogue social & $6 \%$ \\
\hline
\end{tabular}

20. Nous sélectionnons ici les résultats directement en rapport avec notre objet; 10 items étaient proposés et plusieurs réponses étaient possibles.

21. «Pour vous l'intranet c'est»; taux de réponse « oui ». 


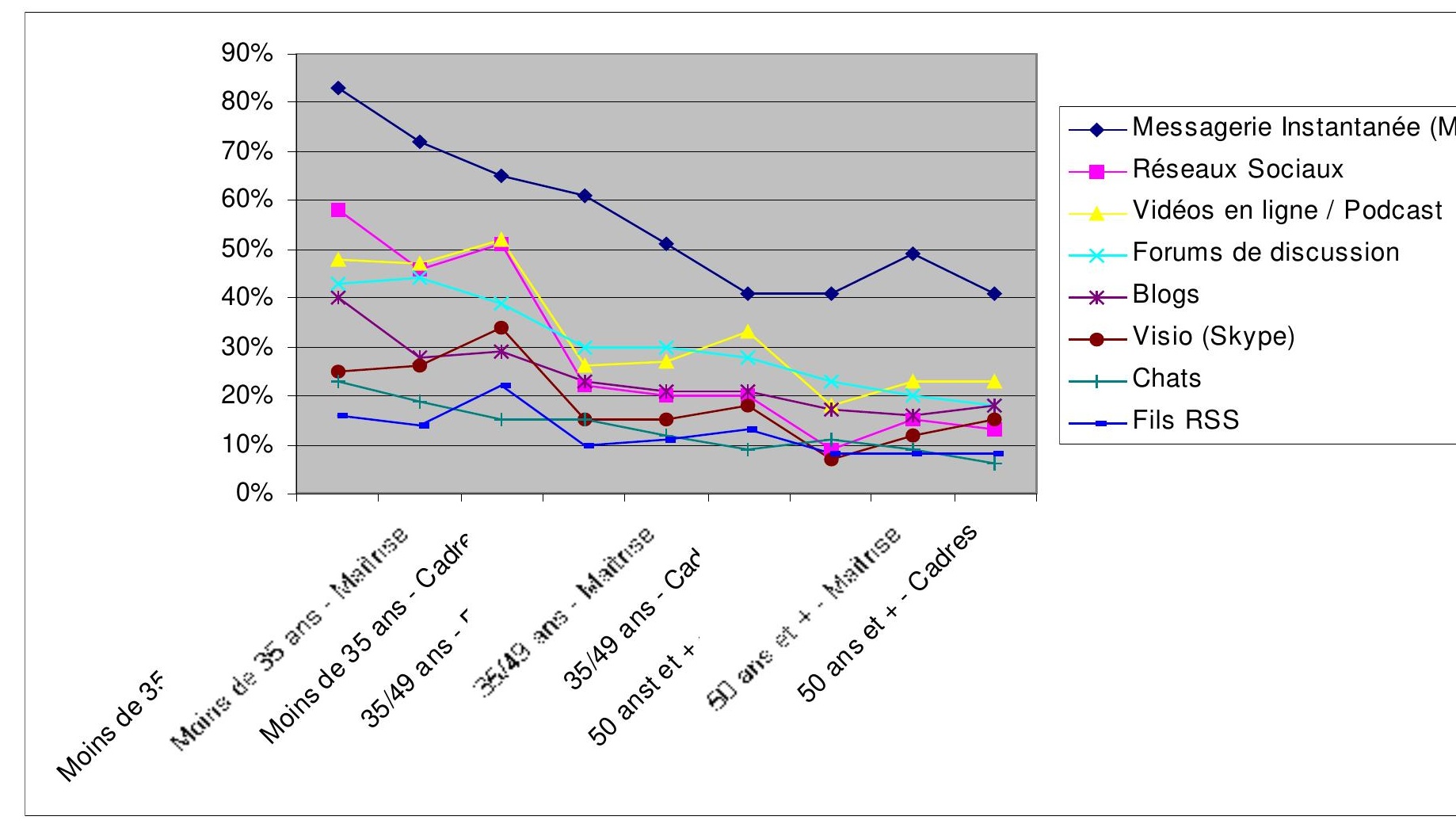

Figure 1. Usages sociaux et interactifs d'internet : variables AGE et STATUT

Par ailleurs, on constate une pratique très élevée, par les salariés, de fonctionnalités interactives et sociales du web (fins professionnelles ou privées confondues) : trois répondants internautes sur quatre sont utilisateurs d'au moins une des ces applications. Sans surprise, on retrouve le niveau d'usage de ces fonctionnalités plus élevé chez les moins de 35 ans et ce, quel que soit le niveau hiérarchique (figure 1). Compte tenu des normes d'avancement de carrière dans cette organisation et de sa structure socioprofessionnelle, il s'avère que les pratiques les plus fortes concernent les salariés issus des premiers niveaux hiérarchiques, donc ayant le statut « agent d'exploitation ».

Sur une tendance identique à la population d'internautes français, les applications synchrones (de type MSN qui était encore très utilisé au moment de l'enquête) sont les plus plébiscitées 22 : $62 \%$ des répondants sont utilisateurs de messageries instantanées. C’est le cas pour $83 \%$ des agents d'exploitation

22. Selon l'etude Ipsos, de septembre 2008, sur les usages d'Internet, $57 \%$ des français seraient utilisateurs de messagerie instantanée. L'enrichissement des fonctionnalités de MSN, plate-forme Windows Live, en fait aujourd'hui l'outil préféré des 18-24 ans, avec $82 \%$ d'utilisateurs, et des 25-34 ans, avec $48 \%$ d'utilisateurs. Devant Facebook : respectivement, $72 \%$ et $46 \%$ des internautes (source : Ifop, 2010). 
de moins de 35 ans avec un écart conséquent par rapport au plus de 50 ans, cadres ou agents d'exploitation (41\% pour ces deux profils).

Second type d'outils le plus utilisé, les réseaux sociaux numériques (RSN) concernent $34 \%$ des répondants : $55 \%$ de la totalité des moins de 35 ans (ce taux est de $58 \%$ pour les agents d'exploitation de moins de 35 ans, et environ la moitié des agents de maitrise et des cadres les utilisent) ; $20 \%$ des 35-49 ans (en fonction du statut, de $22 \%$ à $20 \%$ ); $11 \%$ des 50 ans et plus (de $16 \%$ à $10 \%$ ). Le taux le plus faible se situe chez les agents d'exploitation de plus de 50 ans $(10 \%)$, ce qui confirme que l'âge, mais aussi le type d'emploi occupé, le diplôme, constituent des variables discriminantes (corrélativement, le niveau de rémunération, le non-usage de l'informatique au travail ainsi qu'éventuellement l'absence d'enfants au domicile, seraient à étudier) ${ }^{23}$.

En comparaison, selon l'étude 2009 du Crédoc (année de réalisation de l'enquête), le taux d'utilisateurs de RSN parmi les internautes français était de $33 \%$ en 2009 : $65 \%$ pour les 18-24 ans ; $34 \%$ pour les 25 39 ans ; $10 \%$ pour les 40-59 ans. Les résultats obtenus pour cette organisation suivent donc les tendances nationales, la différenciation en fonction de l'âge, mais l'écart semble s'accentuer par rapport au type de métier occupé : quand pour le Crédoc, $20 \%$ des ouvriers et $23 \%$ des employés sont utilisateurs de RSN, au sein de Angus, le niveau «exploitation » enregistre $40 \%$ de salariés internautes usagers de ces outils (écart de plus de 10 points avec les maitrises et les cadres). L'hypothèse d'un lien entre pratiques socionumériques professionnelles sur internet et faiblesse du positionnement social de l'intranet reste pleinement posée.

Un des problèmes que doit affronter l'organisation étudiée ici, tient au fait que les salariés appartenant au collège Exploitation (premier niveau hiérarchique) ainsi que les plus jeunes, apparaissent comme les plus experts ${ }^{24}$. Cette acquisition de compétences est potentiellement capitalisable, re-territorialisable, en nouveaux professionnalismes par l'entreprise qui doit alors dépasser le postulat, prévalant auparavant, d'une domination des cadres en matière de pratiques info-communicationnelles. De plus, les entretiens réalisés ont permis l'expression d'un ensemble de revendications se rapportant aux performances attendues du dispositif intranet :

- Une prescription fonctionnelle est réalisée par des salariés-experts, dont la montée en compétences s'accompagne d'une exigence accrue vis-à-vis de l'offre TIC (temps de chargement, richesse de l'offre fonctionnelle, performances techniques des outils).

- La création d'espaces adéquats dédiés aux collaborations professionnelles.

- Une prescription sur le moteur de recherche : une référence massive à Google a été identifiée (le modèle Google, même discutable à plusieurs titres, évidemment, s’impose).

23. Selon les résultats de l'étude Crédoc 2009, $92 \%$ des diplômés de l'enseignement supérieur sont internautes alors que moins de 1 sur 3 dans la population non diplômés. Si entre 2004 et 2009, le taux d'accès à Internet des employés à partir du domicile a augmenté de 40 points et de 39 points pour les ouvriers, ce sont les catégories d'actifs les moins connectées (respectivement $74 \%$ et $66 \%$ ). De même, il convient de rappeler qu'en 2009, seuls $34 \%$ des employés et $18 \%$ des ouvriers bénéficiaient d'une connexion internet au travail (48\% pour l'ensemble des actifs.24. Les salariés du statut "exploitation» (salariés de terrain, techniciens, etc.) représentant $55 \%$ des effectifs totaux de l'entreprise Angus; les agents de maitrise (encadrement intermédiaire, chef d'équipe etc.), $28 \%$ des effectifs ; et les cadres, $17 \%$ des effectifs. 
- Une demande de personnalisation : référence est faite notamment à Yahoo ! et à Netvibes.

On voit donc comment les scripts socio-techniques et le référentiel d'internet opèrent ici sur les propres perceptions des salariés-internautes. Cela n'est pas sans conséquences pour la conception des nouveaux dispositifs numériques en organisation et leurs gouvernances. Cet apprentissage TIC qui s'effectue «hors des murs » de l'entreprise, et les compétences ainsi acquises, se déplacent au sein de l'espace professionnel. On assiste alors à une re-territorialisation de ce capital intellectuel et à l'enrichissement de «compétences info-communicationnelles distribuées». Cette performation expérientielle, couplée à une performation machinique, affronte le référentiel des chefs de projets qui y voient une remise en cause de leur propre pouvoir de prescription et de formatage. Cette tension est également explicite dans les débats relayés sur «les risques» d’une soumission des rationalités organisationnelles à ce que certains décrivent comme un «enchantement technologique » portés par les attentes des Digital Natives (Prensky, 2001), qui forment d'ailleurs une "classe » d'usagers bien plus hétérogène qu'il n'y paraît. La perception et le jugement du management vis-à-vis de ces derniers est diverse : pour eux, soit ils constituent une ressource ou un levier d'innovation, soit ils sont, au contraire, évalués en tant que contrainte, ou encore comme un risque de déstabilisation des projets TIC. En résonance, un réseau des responsables intranets 2.0 qualifiait récemment de «mission impossible», le traitement des attentes multiples de la Net Génération tant le retard des politiques intranets en matière de services 2.0 était saillant. En second lieu, à l'instar du cas Angus, c'est la transformation des modèles hiérarchiques qui est mise en jeu. De même, le groupe La Poste s'interrogeait ainsi sur le décalage susceptible d'apparaître entre la communication transversale et spontanée, favorisée par ces outils, et le découpage en silos adapté à une circulation à dominante verticale de l'information qui caractérise les structures formelles de la plupart des organisations ${ }^{25}$. En 2008, on lisait aussi qu'avec l'entrée du web 2.0 dans l'entreprise "Les employeurs et les RH doivent se demander comment ils devront canaliser cette énergie » ${ }^{26}$.

\section{Pratiques expressives et sociales des salariés sur la toile : une polémologie numérique}

Que ce soit dans le cas Angus ou Karina, l'intensité des pratiques socio-numériques est observable directement sur internet. L'étude de la présence en ligne des salariés de Angus a en effet permis d'identifier le développement d'interactions entre collègues, notamment via des forums (l'un d'entre eux revendiquant 10000 membres). De même, en ce qui concerne Karina, nous avons ici affaire à un usage d'internet comme territoire de socialisation et d'expression professionnelles. Nous avons étudié les réseaux et groupes fédérant plusieurs salariés de cette organisation, en prenant en compte des espaces aux fonctionnalités et aux contraintes techniques différentes: forums, blogs collectifs et réseaux sociaux numériques.

Ont été relevés six positionnements principaux caractérisant ces communautés ${ }^{27}$ : voir tableau 2.

25. Appel à recherche, Les réseaux sociaux numériques et leur impact sur l'entreprise; groupe La Poste, 31 janvier 2008.

26. http://www.silicon.fr/fr/news/2008/06/12/le_web_2_0_un_outil_de_gestion_rh.

27. Terme pris au sens de F. Tonnies (1922) faisant une distinction entre Gemeinschaft (communauté), une association d'êtres humains où le groupe est prioritaire par rapport à l'individu, et Gesellschaft (société) où l'intérêt particulier l'emporte sur l'intérêt commun. L'emploi du terme « communauté » fait ici référence à un commun: des idées, valeurs et signes partagés qui établissent la particularité du groupe ; l'identité des membres du groupe est alimentée par cette identité collective. 
Tableau 2. Positionnements des communautés de salariés «Karina » sur internet

\begin{tabular}{|c|c|}
\hline Positionnements & Finalités explicites et pratiques \\
\hline Politique critique & $\begin{array}{l}\text { Critique des politiques de l'organisation; expression de } \\
\text { craintes quant à ses projets, aux politiques RH etc.; média } \\
\text { de communication en contexte conflictuel ; outil de suivi } \\
\text { des grèves (les sites et blogs syndicaux occupent ce } \\
\text { positionnement, mais des collectifs revendiquant leur } \\
\text { autonomie investissent également ce champs). }\end{array}$ \\
\hline Politique solidaire & $\begin{array}{l}\text { Défense de l'organisation et de ses métiers (contre-attaque } \\
\text { faisant suite à des critiques de clients par exemple). }\end{array}$ \\
\hline Veille & $\begin{array}{l}\text { Recherche de sources d'informations générales entre } \\
\text { collègues (sur la concurrence, les dimensions RH, le droit } \\
\text { du travail etc.) ; transmission de documents généraux sur } \\
\text { l'entreprise ; veille de presse etc. }\end{array}$ \\
\hline Identitaire & $\begin{array}{l}\text { Publication et actualisation de «marqueurs sémiotiques » } \\
\text { (jargons, photos, vidéos d'équipement, d'un espace de } \\
\text { travail), volonté de conservation de la «mémoire»du } \\
\text { métier ou de l'organisation, son histoire et ses normes } \\
\text { culturelles. }\end{array}$ \\
\hline Métier/Pratiques & $\begin{array}{l}\text { Echange de savoir-faire et de connaissances en rapport } \\
\text { avec une problématique métier, échange éventuellement } \\
\text { porteur d'un apprentissage mimétique, et pouvant aller } \\
\text { vers une communauté épistémique (création de nouveaux } \\
\text { savoirs) ; entraide sur des sujets très opérationnels } \\
\text { (information sur un nouvel équipement etc.) ou entraide } \\
\text { psychologique (cas de la souffrance au travail). }\end{array}$ \\
\hline Apprentissage/intégration & $\begin{array}{l}\text { Transmission d'une vision métier dans une visée } \\
\text { formative, informations sur le processus de recrutement, } \\
\text { conseils auprès de candidats, auprès de nouveaux } \\
\text { embauchés... }\end{array}$ \\
\hline
\end{tabular}

Au sein de ces communautés, chacune de ces dimensions peut être plus ou moins présente. On a affaire à des espaces hybrides. Par exemple, un forum de discussion de près de 3200 membres et 50000 messages, indique dès sa page d'accueil, son positionnement politique critique et présente une liste de liens vers diverses centrales syndicales (il tient ici un rôle de portail). Il propose également une offre informationnelle de veille environnementale, sociétale, ressources humaines, et enfin, nombre d'échanges portent sur un ensemble de problèmes se rapportant à des procédures métiers. Un autre forum encore (2 100 membres et plus de 55000 messages) plus consensuel quant aux politiques d'entreprise, est orienté « informations RH» à destination de candidats souhaitant postuler au sein de l'entreprise. S'y déroulent aussi de nombreuses interactions exprimant une solidarité entre collègues, une défense des métiers, et 
parfois même de l'organisation et de certaines «valeurs». Enfin, des groupes Facebook (la plupart n'agrégeant que quelques centaines de membres), aux contenus en accès libre, peuvent être utilisés comme médias complémentaires aux sites internet (cas des organisations syndicales), ou comme espace d'agrégation/diffusion d'une mémoire métier (photos d'équipements, vidéos de salariés effectuant leur travail, anciennes affiches ou anciennes communications de l'entreprise). Le positionnement de ces communautés et la richesse des interactions entre les deux types de plateformes sont explicitement conditionnés par le degré d'exposition souhaitée par les participants ou contraint par l'outil utilisé (Facebook semble ici ne pas favoriser de fréquents échanges et de longues discussions). Ainsi, quand sur les forums critiques-politiques le pseudonymat est de rigueur, l'usage d'une identité réelle (nom/prénom et photo, le plus souvent personnelle ou familiale), sur Facebook, est par contre systématisé : l'ancrage du réseau dans un positionnement «vie privée » et l'utilisation d'un compte le plus souvent unique, favorise ainsi un enchevêtrement des territoires privés et professionnels. On connait également les débats associés aux subtilités complexes du paramétrage de son profil public sur le fameux réseau ${ }^{28}$. Nous ne précisons pas ici toutes les analyses effectuées sur ces communautés dont les résultats pourront être développés par ailleurs.

Pour les directions des deux organisations étudiées, cette extimité collective ${ }^{29}$ et des mondes professionnels sur la toile s'accompagne de réflexions convergentes. Pour les responsables de projets TIC et $\mathrm{RH}$, les risques d'intelligence informationnelle, de perte de confidentialité des informations, de veille concurrentielle et sociale est soulevé. Devant faire face à de fortes contraintes commerciales et de gestion des ressources humaines, les acteurs soulèvent également le risque des «chasseurs de tête» et de prospections facilitées de salariés. Enfin, les communautés métiers autonomes s'inscrivent, pour certains responsables, dans un rapport concurrentiel avec l'intranet et avec les stratégies de communication externe (le marché de «l'e-réputation» trouve ici ses débouchés). Dans ce contexte, l'hypothèse de création de « réseaux sociaux numériques » au sein de l'intranet (un Facebook dans sa version entreprise) est vue, par les responsables TIC et Ressources Humaines, comme une opportunité de concurrencer les territoires numériques des salariés, se développant hors des frontières normatives de l'organisation. Dès lors, l'affrontement des processus performatifs met au jour les dimensions bio-techno-politiques attachées à un désir de contrôle sur les modes d'individuations professionnelles et de «maitrise ichnologique » (Merzeau, 2009). Ces traces, qui collectent des cascades de trans-formations toujours plus élaborées, ont un caractère éminemment paradoxal puisque plus l'informatisation (numérisation) se répand, plus nous pouvons pister ce qui nous attache les uns aux autres (Latour, 1998). Ainsi, les pratiques socio-numériques des salariés imposent aux entreprises une réflexion non seulement stratégique, mais également éthique sur la liberté d'expression, le contrôle et l'exploitation des données, d’un territoire numérique organisationnel étendu. $\begin{aligned} & \text { 28. Voir, par exemple, l'article du New York. Times, } 12 \text { mai 2010, indiquant qu'à cette date, FB proposait } 50 \text { critères de } \\ & \text { renseignements }\end{aligned}$ et $170 \quad$ options com/2010/05/13/technology/personaltech/13basics.html?pagewanted=print.

29. En écho au désir d'extimité comme exposition d'un soi intime (Tisseron, 2001)

30. Dans ce cas, le licenciement de salariés, ayant exprimé une critique vis-à-vis d'un supérieur, celui-ci ayant eu accès aux échanges en tant qu'ami « de second niveau » (les amis des amis des salariés en question avait accès à leur Mur), a été jugé comme fondé. Un profil $\mathrm{FB}$ « ouvert» est alors considéré comme un espace public et un profil strictement réservé « aux amis » de premier niveau, comme un espace privé. Reste toutefois posée la problématique des associations en chaine. Rappelons que selon l'article L2281-3 du code du travail, «les opinions que les salariés, quelle que soit leur place dans la hiérarchie professionnelle, émettent dans l'exercice du droit d'expression, ne peuvent motiver une sanction ou un licenciement». Les restrictions sont relatives aux propos injurieux et diffamatoires ou encore justifiées par la nature de la tâche à accomplir. 
En la matière, il s'agit évidemment de suivre l'évolution du cadre juridique dont on connait l'itération mais aussi parfois les paradoxes. Le jugement rendu par le tribunal des Prud'hommes de Boulogne Billancourt en 2010 a institué de facto une obligation de maittrise des subtilités des paramétrages de Facebook et de leurs évolutions ${ }^{30}$, considérant au passage que la sphère «des amis d'amis » était un espace public. Mais, en 2011, la Cour d'appel de Besançon fait la distinction entre les publications Facebook ouvertes à tous et les éléments du "mur" dont l'accès est limité aux seuls contacts et qui dans ce cas, devait être considéré comme un espace privé. Enfin, la Cour de cassation semble avoir tranché en 2013 : un profil Facebook n'est pas un lieu public si le titulaire de celui-ci n'en n'a autorisé l'accès d'un nombre très restreint "d'amis » 31. Etant peu nombreux et agréés entre eux, auteur et lecteurs sont liés par une « communauté d'intérêt » et les publications des salariés ne seraient alors plus susceptibles de faire l'objet de poursuites pour diffamation ou injure publique. Ces distinctions ténues peuvent également être soumises à divers débats sur les sphères professionnelles/privées au sein d'une entreprise : selon quel régime de visibilité et quelle économie relationnelle, les réseaux sociaux internes (qui, dans certains cas, ne relèvent pas des outils de travail obligatoires) se déploieront-ils?

\section{Conclusion}

Cette analyse de la construction des stratégies numériques en organisation a permis d'initier un questionnement des processus d'innovation à partir du phénomène performatif en mettant en évidence des configurations hétérogènes, dynamiquement enchevêtrées. Sans prétendre à l'épuisement des enjeux théoriques, méthodologiques et des phénomènes qui se présentent là, nous avons exposé dans cet article, un moment d'une réflexion plus générale, qui porte sur les narratiques et les dispositifs concrets au sein desquels se construit et se développe une politique managériale. La poursuite de ce travail soulève diverses difficultés quant à l'observation de l'accomplissement des processus de formatage et requiert des enquêtes ethnographiques complexes, en intégrant également la question des temporalités caractéristiques des processus d'innovation en cours, des asynchronies et diachronies de l'ère numérique (Stiegler, 2008). Cela implique que soient prises en considération toutes les lignes de performation/création dès les microprocessus d'innovation, les micro-devenirs, dont l'agencement favorise plus ou moins la distribution. Plus globalement, cette approche invite à prendre en compte les performations agonistiques et les enjeux bio-politiques accompagnant le formatage des organisations et des univers existentiels au travail.

\section{Bibliographie}

Akrich M., «La construction d'un système socio-technique : esquisse pour une anthropologie des techniques », Anthropologie et Sociétés, 13(2), 1989, p. 31-54.

Akrich M., «Comment décrire les objets techniques ? », Techniques et culture n 9, 1987, p. 49-64.

Allard L., «Blogs, podcasts, Tags, Mashups, Locative Médias : le tournant expressiviste du web in 2.0 », Culture numérique, cultures expressives, revue MédiaMorphoses, $\mathrm{n}^{\circ}$ 21, septembre 2007, p. 57-68.

${ }^{31}$ Le 10 avril 2013, les juges de la Cour de cassation ont déclaré qu'une employée, plaidant sur sa page Facebook et sur son compte MSN pour "l'extermination des directrices comme la (sienne)" et des "patronnes (...) qui nous pourrissent la vie", ne pouvait pas se voir reprocher d'avoir tenu ces propos publiquement puisqu'ils n'étaient accessibles qu'à ses "amis" ou "contacts".L'arrêt de la Cour de Cassation :

http://www.courdecassation.fr/jurisprudence 2/premiere chambre civile 568/344 10 26000.html 
Austin J. L., Quand dire c'est faire, Paris, Le Seuil, 1970.

Bourdieu P., Ce que parler veut dire, Paris, Fayard, 1982.

Boyd D., Ellison N., "Social Network Sites: Definition, History, and Scholarship", Journal of Computer-Mediated Communication, vol. 13, $\mathrm{n}^{\circ} 1,2007$.

Callon M., The Laws of the Markets, Blackwell, Oxford, 1998.

Callon M., Latour B., "“Tu ne calculeras pas!” ou comment symétriser le don et le capital» in Alain Caillé (sous la direction de) Le capitalisme aujourd'bui, Revue du MAUSS, nº 9, La Découverte, Paris, 1997, p.45-70.

Callon M., "Réseaux technico-économiques et irréversibilités », In Boyer R., Chavance B., Godard O. (sous la dir. de), Les Figures de l'irréversibilité en économie, Paris, Editions de l'École des Hautes Études en Sciences Sociales, 1991.

Callon M., Latour B., La science telle qu'elle se fait, Paris, La Découverte, 1991.

Carmes M., Galibert O., «L'enchevêtrement des territoires numériques inter-intranet : un questionnement des déterritorialisations/re-territorialisations des pratiques salariés ", Pour une approche communicationnelle de l'individu au travail, Revue Communication \& Organisation, GREC /O Université de Bordeaux 3, nº 36, 2010, p. 191-223.

Carmes M., «Les activités de contribution dans la politique intranet d'un Conseil Général: d'une injonction institutionnelle à une sémio-politique de la "transversalité », Revue Études de Communication, L'activité aux prises avec des systèmes ou dispositifs d'information, $\mathrm{n}^{\circ}$ 33, Université Lille 3, 2009, p 19-39.

Carmes M., «L'organisation peut-elle s'inventer par les scripts ? Performations et actualisations dans les agencements Intranet", Actes du colloque Les dispositifs de médiation organisationnelle, technologique et symbolique dans la communication des organisations, laboratoire I3M Université de Nice Sophia Antipolis, 5 décembre 2008, p. 260-268 http://hal.archives-ouvertes.fr/hal-00411233/fr/.

Cochoy F., Giraudeau M., McFall L (dir), "Performativity, Economics and Politics : an overview”, Journal of Cultural Economy, vol. 3, 2010.

Coreen F., "Ventriloquie, performativité et communication, Ou comment fait-on parler les choses », in Un tournant performatif ? Retour sur ce que «font» les mots et les choses, Réseaux 2010/5 (n 163), 2010.

Coutant A, Stenger T, «Processus identitaire et ordre de l'interaction sur les réseaux socionumériques », Les enjeux de l'information et de la communication août 2010 [ en ligne ] http://w3.ugrenoble3.fr/les enjeux/2010/Coutant-Stenger/index.html. , consulté le 3/12/2010

D'Almeida N., Les promesses de la communication, Paris, PUF, 2001.

De la Broise P, et Grosjean S, «Introduction », in Normes et écriture de l'organisation, Études de communication, 34/2010, [En ligne], mis en ligne le 14 septembre 2010. URL : http://edc.revues.org/index1752.html.

Deleuze G., Dialogues, avec Claire Parnet, Paris, Flammarion (coll. «Champs »), 1977, 2e éd. 1996.

Deleuze G., Guattari F., Mille Plateaux - Capitalisme et schizophrénie 2, Paris, Les éditions de Minuit, coll. «Critique », 1980.

Denis J., «Préface : Les nouveaux visages de la performativité », in Performativité : Relectures et usages d'une notion frontière, Études de communication, 29/2006, [En ligne] le 19 janvier 2009. URL: http://edc.revues.org/index344.html.

Denis J., «Les règles et leur disponibilité », Journées Écritures normées, écritures normatives, MESHS, Lille, 2-3 avril 2009.

Fraenkel B., «Actes écrits, actes oraux : la performativité à l'épreuve de l'écriture », in Performativité : Relectures et usages d'une notion frontière, Études de communication (29), 2006, p. 69-93.

Grosjean M., Lacoste M., «L'oral et l'écrit dans les communications de travail ou les illusions du «tout écrit»», Sociologie du travail, XL (4), 439-465, 1998. 
Gryspeerdt A, Marion P. (coord), Esthétique des organisations, Revue Recherches en Communication, n¹7, 2002.

Guattari. F, «Les systèmes d'interfaces machiniques », Terminal, n52, 1991.

Latour B, Changer la société, refaire de la sociologie, La Découverte, 2006.

Latour B., Les Microbes : guerre et paix, suivi de Irréductions, La Découverte 1984, (réed 2001).

Latour B., Hermant É., Paris ville invisible, Paris, Éditions Les empêcheurs de penser en rond, La Découverte, 1998.

Lazzarato M., Le « pluralisme sémiotique » et le nouveau gouvernement des signes, Hommage à Félix Guattari, Institut Européen pour des politiques culturelles en devenir, 2006.

Levy P., Qu'est ce que le virtuel, Paris, La Découverte, 1995.

Licoppe C. (dir), L'évolution des cultures numériques, Fyp Eds, 2009.

Lyotard JF., La condition post-moderne, Éditions de Minuit, 1979.

Merton K., Social Theory and Social Structure, Eléments de théorie et de méthode sociologique, rééd. Armand Colin, (1949) 1997.

Merzeau L., « De la surveillance à la Veille », Cités, nº 39, Presses Universitaires de France, 2009.

Millerand F., Proulx S., Rueff J. (dir), Web social, Mutation de la communication, Presses de l'Université du Québec, 2010.

Moatti, « Représentation de soi et identité numérique. Une approche sémiotique et quantitative de l'emprise culturelle du web $2.0 »$, Revue Réseaux, n 154, 2009.

Muniesa F., Callon M., La performativité des sciences économiques, Papiers de recherche du CSI, CSI Working papers Series, $\mathrm{n}^{\circ} 10,2008$.

Noyer J.M., Juanals B., (Dir), Technologies de l'Information et Transformation des Intelligences Collectives, Hermès-Lavoisier, 2010.

Noyer J.M., Carmes M., «Régimes d'interfaces : vers une sémiopolitique des espaces numériques organisationnels », in Broudoux E. et Chartron. G, Enjeux politiques du document numérique, $3^{e}$ conférence Document Numérique et Société, eds ADBS, 2010, p. 195-216.

Noyer J.M., «De l'extension illimitée des études d'usages : quelques remarques », Actes du Colloque Pratiques et usages organisationnels des sciences et technologies de l'information et de la communication, Université de Rennes 2, 7-9 septembre 2006, p. 36-39.

Noyer J.M., «Les temporalités militaires et stratégiques aujourd’hui », Les Cabiers du numérique 1/2002, vol. 3, p. 125137.

Palpacuer F., Leroy M., Naro G., (coord.), Management, mondialisation, écologie : regards critiques en sciences de gestion, Hermès-Lavoisier, 2010.

Stiegler et al., Les réseaux sociaux, FYP, 2010.

Stiegler B., Economie de l'immatériel et psychopouvoir, Entretiens avec Philippe Petit et Vincent Bontems, Paris, Mille et Une Nuits, 2008.

Stiegler B., La technique et le temps, La désorientation, Galilée, 1996.

Tisseron S., L'intimité surexposée, (2001) 2003, Ramsay.

Tönnies F., Communauté et société. Catégories fondamentales de la sociologie pure. Introduction et traduction de J. Leif. Titre allemand original: Gemeinschaft Und Gesellschaft. Paris, Les Presses universitaires de France, (1922) 1977. 